\title{
Competencias educativas en la modalidad virtual docentes, discentes, y el mundo laboral
}

\author{
Educational competences in the virtual modality teachers, \\ students, and the world of work
}

Jennifer Wall Lumseyfai (iD Diana Marcela Toro (iD Beatriz Elizabeth Nagari $\square$

Universidad Metropolitana de Educación, Ciencia y Tecnología. Ciudad de Panamá, Panamá

Recibido: 05/08/2021 Revisado: 20/09/2021_Aceptado: 22/09/2021 Publicado: 31/10/2021

\section{RESUMEN}

El presente artículo pretende reflexionar sobre el desarrollo de competencias en la modalidad virtual del siglo XXI a través del estudio de docentes, estudiantes y la fuerza laboral una vez completada la educación formal. Este ensayo busca interpretar cuáles de las competencias son necesarias para que los docentes enseñen de manera efectiva incluyendo las competencias relacionadas con la información previa que los docentes necesitan para una enseñanza y planificación efectivas, las competencias para la enseñanza del aprendizaje, así como el valor y evaluación de la enseñanza y el aprendizaje. En segundo lugar, este ensayo investiga las competencias del discente en el mundo virtual incluyendo: competencia pedagógica (aprender a aprender), comunicación, competencia psicológica (aprendizaje socio-emocional), competencia digital y competencia investigativa. Finalmente, el ensayo conecta las capacidades tanto de los profesores como de los estudiantes para discernir las competencias que son esenciales para la incorporación de nuestros aprendices a la fuerza laboral actual. Estas habilidades incluyen liderazgo, trabajo en grupo, educación y aprendizaje digital. Juntos, estos tres enfoques permiten un camino claro para aquellos que están enseñando cómo aprender, aquellos que están aprendiendo y aquellos que están aplicando su aprendizaje en un entorno virtual. Palabras Clave: Competencias virtuales, aprendizaje, futuro, enseñanza.

\section{ABSTRACT}

This article aims to reflect on the development of skills in the virtual modality in the 21 st century through the study of teachers, students, and the workforce after formal education is complete. This essay seeks to interpret which of the competencies are necessary for teachers to teach effectively including the competencies necessary for teaching, the prior information teachers need for effective teaching and planning, the competences for teaching learning as well as the value and evaluation of teaching and learning. Secondly, this essay 
investigates the competencies of the students in the virtual world including pedagogical competence (learning to learn), communication, psychological competence (socio-emotional learning), digital competence and investigative competence. Finally, the essay connects the capacities of both teachers and students to view the competencies that are essential for today's workforce. These skills include leadership, group work, education, and digital learning. Together these three focuses allow for a clear path from those who are teaching how to learn, those who are learning, and those who are applying their learning in a virtual setting. Keywords: Virtual competencies, learning, future, teaching.

\section{INTRODUCCIÓN}

El presente artículo se desarrolla a partir de una revisión bibliográfica, acerca de cómo el aprendizaje en la modalidad virtual incide en la promoción del desarrollo de competencias, las cuales son necesarias para la preparación de los educandos teniendo en cuenta las exigencias laborales propias del siglo XXI. Cuando se habla de competencias, lo primero que viene a la mente es el bien que han causado al proceso educativo, haciéndolo más coherente y creando una conexión "real" entre educación y fuerza laboral, así como el beneficio de proveer experiencias y prácticas de trabajo reales para los aprendices. Sin embargo, es imprescindible mencionar de igual modo los desafíos o retos que las mismas pueden causar si no hay una estructura o un seguimiento de políticas actualizadas que respondan a los cambios y situaciones propias del momento histórico que se vive.
La presente reflexión y crítica nace teniendo en cuenta que el desarrollo de competencias al igual que el uso de herramientas tecnológicas hace parte de la vida de los jóvenes de la Generación $Z$, es decir los nacidos a partir del año 2,000, desde su misma concepción. La relevancia de este tema se da con mayor validez dado que son ellos quienes están llenando los salones de los centros educativos de todos los niveles en la actualidad y quienes se están formando para convertirse en la siguiente fuerza laboral del siglo XXI.

Es por esto que se hace necesaria la transformación de la educación actual mediante la activación de recursos gubernamentales que permitan la capacitación de los docentes en nuevas tecnologías de la información y la comunicación, la creación y apoyo financiero de grupos de investigación educativa que desarrollen nuevas tendencias y al-

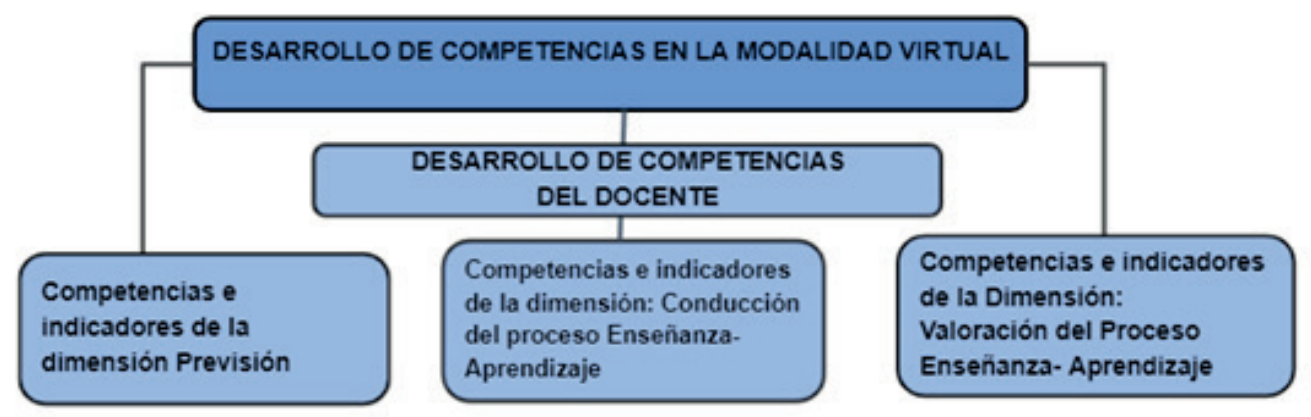

Figura 1. Competencias Requeridas por los Docentes. 
Competencias educativas en la modalidad virtual docentes, discentes, y el mundo laboral

ternativas de aprendizaje desde la aplicación en el aula y la provisión de recursos tecnológicos que permitan tanto a educandos como a educadores pasar de la teoría a la práctica.

Ante los hechos ya conocidos, y los retos que se presentan en el Siglo XXI para la educación y el desarrollo, la educación virtual se muestra como una ventana oportuna y pertinente capaz de hacer llegar la formación a cualquier nivel educativo y prácticamente a cualquier lugar del mundo con la tecnología disponible en la actualidad. La educación virtual debe considerarse en el Siglo XXI un complemento ideal para la educación tradicional. El creciente desarrollo de la misma, la necesidad de aprender a lo largo de la vida y actualizar conocimientos, así como la búsqueda del perfeccionamiento profesional en una sociedad con nuevas oportunidades, pero mayores demandas, hacen de la educación virtual el medio ideal para la adquisición de competencias en el nuevo panorama mundial. Para ello resulta fundamental la participación docente en línea con competencias estratégicas para la enseñanza y evaluación de la docencia en ambientes virtuales de modo que les ayude a fortificar e instrumentar mejoras, que se alineen a la realidad de los sistemas de educación superior. Bonilla- Romeu, (2009 p.138) expuso que todo esfuerzo por iniciar programas de educación virtual, debe estar fundamentado en los principios de calidad institucional y aquellos establecidos por los organismos acreditadores correspondientes. Mientras que Ramírez-Montoya, M. (2019) mencionó que la experiencia de utilización de tecnología en los sistemas educativos de América Latina y el Caribe en los últimos veinte años, ha mostrado poco efecto en la calidad de educación. Para ello se han elaborado tres pasos estructurados que deben perseguir los docentes; la primera competencia es de planificación y preparación previa a la enseñanza, la segunda competencia indica la conducción del proceso enseñanza- aprendizaje, la tercera competencia muestra la valoración y evaluación del proceso enseñanza- aprendizaje.

\section{Competencias de Planificación Previas a la Enseñanza}

Es la organización sistemática de los elementos necesarios y del proceso de enseñanza y aprendizaje, secundaria a la respuesta a la modalidad de enseñanza online. Incluye el trabajo preliminar del docente para definir el programa del curso y la sesión de capacitación en relación a la competencia por negligencia del alumno, por lo que incluye la especificación de las condiciones materiales y operativas necesarias para sustentar el proceso de enseñanza, aprendizaje y su evaluación. García B. et al. (2018) realizaron un estudio descriptivo en el que emplearon equipos de debate y técnicas de análisis de contenido, las mismas que los llevó a la definición de las competencias previas a la enseñanza, ellos reiteraron la importancia de tener una competencia en el planteamiento del enfoque de la asignatura donde se establecen las metas generales y se relacionan con el plan de estudio. Como segunda competencia indicaron la necesidad de que el docente planee la asignatura de una manera específica que le ayude a definir el enfoque didáctico del curso y como última competencia previa a la enseñanza, el docente debe diseñar experiencias de aprendizaje donde se provea alternativas de estrategias didácticas para ayudar a los alumnos. En ese sentido, Torrealba et al. (2020) consideran, que las tecnologías de la información proveen estrategias pragmáticas para el desarrollo de actividades académicas, generando la interacción, destrezas comuni- 
Competencias educativas en la modalidad virtual docentes, discentes, y el mundo laboral

cativas, dinámicas en los contenidos de modo que contribuyan al adecuado desarrollo de los contenidos. Rodríguez y Barragán (2017 p.1) concuerdan que si los estudiantes son incentivados al Entorno Virtual de Aprendizaje (EVA), ellos podrán desarrollar sus destrezas y de consecuencia su rendimiento académico.

\section{Competencias del Proceso de Enseñanza- Aprendizaje}

Alamri y Tyler-Wood, (2017) mencionan de los resultados de varias investigaciones realizadas que de la forma en que un docente interactúa y basa su presencia en línea puede tener un efecto significativo en la experiencia de aprendizaje general de los estudiantes, ya que la calidad del aprendizaje es mayor cuando existe una presencia física (virtual) y emotiva del profesor, lo cual contrasta con aquellas situaciones en las que los estudiantes interactúan con sus compañeros sin la participación del profesor. En el estudio de García B. et al. (2018) se definió al proceso de Enseñanza- Aprendizaje cuando el profesor gestiona el progreso del aprendizaje; provee una introducción del curso, materiales y acceso a las TIC 's, propicia conexión de ideas, propone escenarios alternativos, aclara ideas, e impulsa a los estudiantes a pensar de una manera crítica. Como segunda competencia el docente encamina a los estudiantes a la reciprocidad didáctica de modo que los instruye a acrecentar su motivación y sus expectativas
Nagari, B y cols.

y la última competencia se vio la importancia de agregar las formas de comunicación ya sean de conocimientos o sentimientos. En este mismo estudio de García B. et al. (2018) los alumnos han reportado que la presencia del profesor era intrínseca, no sólo como una presencia física-virtual e inclusive para compartir los conocimientos profesionales sino también en lo relacional donde los valores de identidad, creencias, integridad, pensamientos y emociones eran compartidos. Es decir, los alumnos han aludido a la necesidad de contar con instructores virtuales que proporcionasen una presencia personal- relacional.

\section{Competencias de Valoración y Evaluación de Enseñanza- Aprendizaje}

Contempla los mecanismos y estrategias para la evaluación de las metas, la acreditación de la materia, la satisfacción de las expectativas del propio profesor y de los alumnos, así como la valoración del impacto personal de la experiencia didáctica en línea. La atención por la evaluación de materiales en el marco de la literatura relacionada con la docencia, la organización escolar y la propia tecnología educativa es un tema con una intrínseca trayectoria de investigación y de reflexión por Rodríguez Rodríguez \& Álvarez Seone, (2017). Otro tema diferente es que este grado de reflexión ha tenido sus consecuencias más directas en la práctica educativa. De hecho, gran parte de la investigación reciente parece poner

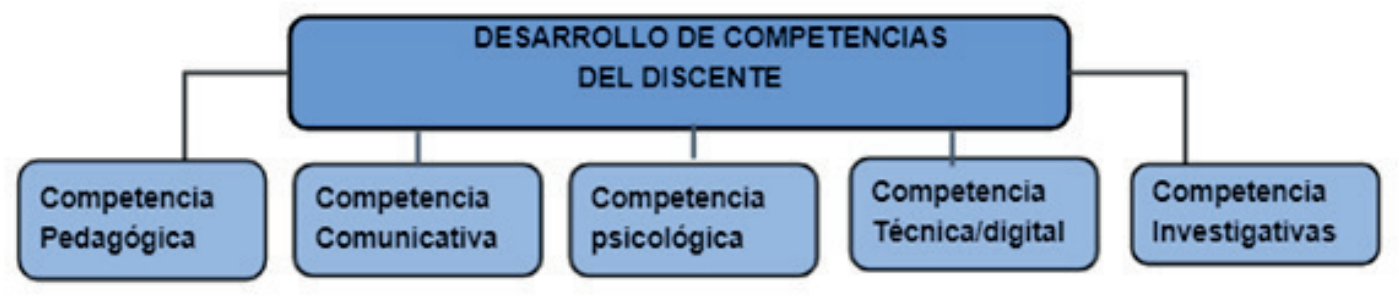

Figura 2. Desarrollo de Competencias del Discente 
Competencias educativas en la modalidad virtual docentes, discentes, y el mundo laboral

de relieve el importante grado de desconocimiento que existe actualmente sobre los modelos y guías para la evaluación de materiales, tanto en documento impreso como digital, y su uso en los sistemas educativos. Rodríguez Regueira y Rodríguez Rodríguez, (2016).

\section{Competencia Pedagógica (Aprender a Aprender)}

La llegada del siglo XXI trajo consigo una serie de cambios a nivel global en las distintas áreas de desarrollo de los seres humanos, no es una sorpresa entonces que el punto de partida de estos cambios haya sido la educación. Se hizo necesario re pensar la educación y crear nuevas alternativas que estuvieran a la altura de los cambios que esta nueva era traía consigo. Como lo describen Aristizábal Montes et al. (2016), la llamada Sociedad del Conocimiento cambió la visión de una educación puramente académica, a una que preparara a sus pupilos para las labores propias de los trabajos a los que se enfrentan, las habilidades clásicas quedaron de lado y se generaron expectativas de nuevas competencias propias de las exigencias del mundo laboral moderno, siendo Aprender a Aprender probablemente la más importante de ellas dentro de estas competencias. Los aprendices de hoy y fuerza laboral a futuro requieren saber cómo obtener la información y procesarla adecuadamente para transformarla en nuevo saber, es decir en nuevas ideas y formas de solucionar problemas. Es por esto que en la actualidad los empleadores prefieren a un empleado novato con aspiraciones de continuar aprendiendo y que tiene un conocimiento más holístico y diverso de su área de trabajo, por encima de uno que presentó excelencia académica, pero se ciñó al trabajo de una sola área, sin experimentar otras posibles alternativas o complementos para la misma, quedándose "estancado" en su proceso de conocimiento competencial.
Nagari, B y cols.

Es necesario que en el proceso de Aprender a Aprender el estudiante participe en una serie de etapas que le permitan transformarse en un individuo más independiente y consciente de su propio aprendizaje, identificando de esta manera sus fortalezas y de igual manera las competencias que requieren una mayor expansión del conocimiento o una práctica más profunda, lo que a largo plazo le permitirá estar mejor preparado para el mundo laboral actual; Aristizábal Montes et al. (2014) proponen que "Aprender a aprender consiste en utilizar de manera adecuada estrategias de aprendizaje cognitivas y metacognitivas para ser más eficientes aprendiendo", de igual manera coinciden en que existen unas características generales que representan las necesidades propias del Aprender a Aprender como son:

1.Metacognición, la cual propone una reflexión individual del discente alrededor de su propio aprendizaje; esto implica según Valenzuela (2019), la participación activa, consciente, intencional y estratégica del aprendiente.

2.Estrategias de estudio de acuerdo con el tema abordado, como lo explica Vera Sagredo (2019) múltiples autores han coincidido en afirmar que las estrategias de aprendizaje son habilidades cognitivas complejas las cuales le permiten al aprendiz desarrollar procesos mentales que les permitan reconocer, entender y adoptar la información requerida para su proceso de aprendizaje.

3.Contar con un asesor/ guía, al respecto Arteaga et al. (2016) explican que es necesario permitir que el estudiante sea el protagonista de su proceso de aprendizaje en el cual él realice una búsqueda reflexiva del conocimiento que no tiene mientras recibe una orientación que le ayude a 
Competencias educativas en la modalidad virtual docentes, discentes, y el mundo laboral

identificar los aspectos en los cuales requiere mayor trabajo o aquellos que le hacen falta.

4.Práctica continua, la cual es la base del mantenimiento y fortalecimiento de las competencias adquiridas a largo plazo, tal y como lo explican Ríos Muñoz \& Herrera Araya (2017) "Las competencias adquieren sentido en la acción y práctica a partir de la experiencia”.

\section{Competencia Comunicativa}

La educación por competencias presenta al estudiante en la modalidad virtual verdaderos desafíos para el proceso de aprendizaje en cuanto al desarrollo de su competencia comunicativa, por un lado, los estudiantes se han tenido que ver enfrentados a nuevos escenarios de comunicación virtual a raíz de la pandemia por COVID-19 los cuales no son los más óptimos, pero, que dan una oportunidad de solventar la crisis educativa actual mediante nuevos y diferentes escenarios de comunicación oral. Por otro lado, si bien es cierto que la comunicación es principalmente escrita, también es cierto que la misma debe estar compuesta de argumentos y contra argumentos que posean bases teóricas relevantes y que además se encuentren descritas en un lenguaje coherente, cohesivo y respetuoso de la audiencia para la que se produce. Estos aspectos pueden presentar dificultades para aquellos discentes para quienes la competencia comunicativa tanto oral como escrita no es innata, como lo explican Valdez-Esquivel \& Pérez-Azahuanche (2021), es evidente que algunas personas presentan mayores dificultades para comunicarse que otras, ¿significa esto entonces que las que tienen mayor habilidad en este proceso no necesitan ningún tipo de preparación?, por el contrario, estas habilidades deben ser practicadas constantemente para alcanzar su mayor potencial y en el caso opuesto la ejercitación permitirá mostrar
Nagari, B y cols.

progreso durante el proceso de aprendizaje.

Es importante recordar que las competencias comunicativas en ambientes presenciales y virtuales son y seguirán siendo la necesidad fundamental-social por excelencia de los seres humanos, pues éstas son las que producen y permiten la interacción entre grupos interdisciplinarios, la exposición de ideas y por último la toma de decisiones conjuntas en pro de las diferentes dimensiones de la sociedad a nivel global; al respecto Morales Mantilla (2011) describe que, según el ICFES (Instituto Colombiano para el Fomento de la Educación Superior) existen tres competencias básicas que se deben potenciar dentro de la dimensión comunicativa a saber Interpretación, Proposición y Argumentación, explica además que en ambientes virtuales las competencias comunicativas poseen un sentido y relevancia especiales para el aprendiz y es justamente por esto que las mismas deben promoverse de manera contextualizada para asegurar el éxito formativo en la modalidad virtual.

\section{Competencia Psicológica (Aprendizaje Socio-Emocional)}

$\mathrm{Si}$ bien es cierto que preparar a las generaciones presentes para los futuros retos del mundo globalizado es una meta continua de la educación, también es cierto que la más importante de estas competencias deberá ser la de aprender a cuidar de sí mismos tanto física como mentalmente. No serviría de nada tener una planta sin tener tierra, tener computador sin tener batería, tener el cuerpo sin tener el cerebro. Es por esto que los gobiernos a nivel mundial se han puesto en la tarea de desarrollar en los estudiantes desde edades tempranas habilidades socio emocionales que les brinden a nuestros aprendices, la resiliencia necesaria para afrontar los retos laborales del siglo XX, así lo enfatizan Guevara Benitez et al. (2020) 
Competencias educativas en la modalidad virtual docentes, discentes, y el mundo laboral

cuando explican las ideas presentadas por la Organización para la Cooperación y el Desarrollo Económico (OCDE, 2015), quienes describen la violencia, el bajo desempeño escolar, y los problemas emocionales y de salud mental como las principales causas de delincuencia y suicidio en países latinoamericanos, sin descartar que estos problemas también se presentan en países más desarrollados como los Estados Unidos. Es por esto que a nivel mundial se está promoviendo el desarrollo de habilidades socio emocionales en todos los niveles educativos, sabiendo que estas mejoran la percepción personal, la calidad de vida, la reducción de la violencia y por último el clima social de los ciudadanos del planeta.

\section{Competencia Digital}

Sin lugar a dudas la competencia digital es uno de los requerimientos básicos con los que cualquier aprendiz del siglo XXI debe contar, tal y como lo explica el Instituto Nacional de Tecnologías Educativas y Formación del Profesorado (2017, p. 12) "puede definirse como el uso creativo, crítico y seguro de las tecnologías de información y comunicación para alcanzar los objetivos relacionados con el trabajo, la empleabilidad, el aprendizaje, el tiempo libre, la inclusión y la participación en la sociedad". Es así como, la competencia digital no se refiere meramente al uso de un computador y sus programas, si no al aprovechamiento de este recurso para la investigación, análisis, discusión y distribución de la información que allí se da, generando cambios en los distintos niveles de la sociedad de manera local, regional, nacional e internacional.

\section{Competencia Investigativa}

Las competencias investigativas según George Reyes et al. (2019) son habilidades de indagación que permiten al educando la resolución de problemas referentes al asunto que se esté tratando, esta actividad se realiza mediante el uso intencional de procesos cognitivos, los cuales incluyen la búsqueda, recuperación, generación, organización, sistematización y análisis de información. En la actualidad las competencias investigativas se encuentran directamente ligadas al uso de las Tecnologías de la Información y la Comunicación (TIC), dado a que la mayoría de los educandos en edad estudiantil de cualquier nivel educativo nacieron o crecieron en la era de la tecnología y por ende, no conocen un mundo sin ella, al respecto Reiban (2018) citado por George Reyes et al. (2019, p. 68) asegura que "las TIC posibilitan el desarrollo de competencias estrechamente vinculadas con el proceso de investigación y con los productos parciales que necesitan generarse durante el trayecto formativo de los estudiantes".

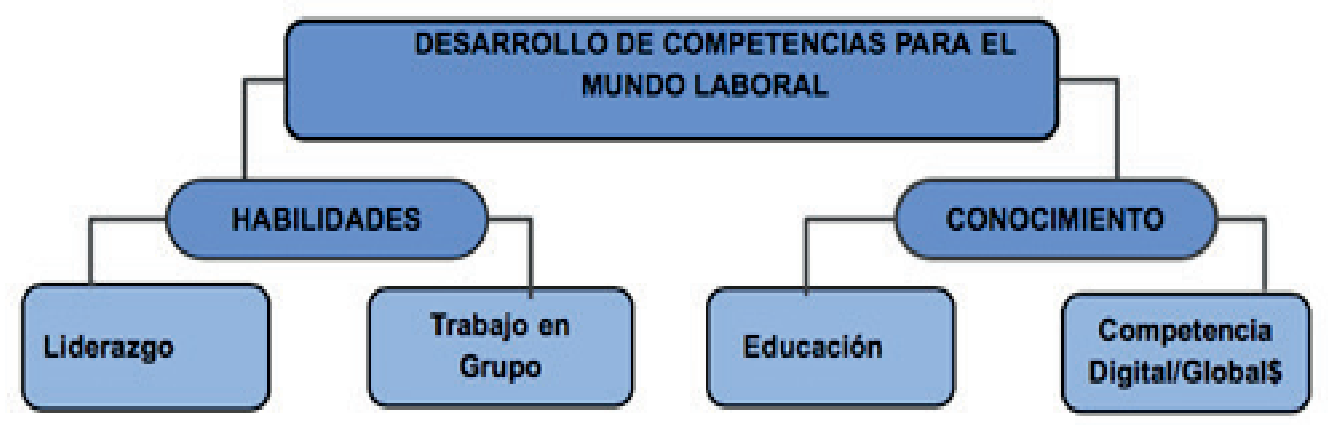

Figura 3. Competencias de Integración al Mundo Laboral 
Competencias educativas en la modalidad virtual docentes, discentes, y el mundo laboral

Las competencias que se esperan cuando alguien ingresa a la fuerza laboral son similares a las competencias que se esperan de profesores y estudiantes. Las expectativas de comprensión y saber hacer el trabajo, aplicación de habilidades, liderazgo, colaboración en grupos y resolución de problemas. Estas habilidades son esenciales para la fuerza laboral a nivel local y global y se pueden encontrar dentro del sistema educativo. Aprender, saber aprender y adaptarse a menudo se asocian con los niños en edad escolar dentro del entorno del aula, sin embargo, incluso como adultos, no dejamos de necesitar esta información crítica. Esto es evidente en las principales características y competencias necesarias para ser un miembro valioso de la fuerza laboral y del mundo. Según la agenda de UNESCO 2030, su primera acción es "Educación inclusiva de calidad y aprendizaje permanente para todos" (UNESCO, 8) y su octavo objetivo es "equipar a jóvenes y adultos con las habilidades necesarias para el empleo, el trabajo decente y el espíritu empresarial". Demostrar que las habilidades y competencias van más allá del aula y llegan a la fuerza laboral.

La habilidad de conocer el trabajo y saber cómo aprender nuevas habilidades es un componente crítico de la fuerza laboral. Según Zermeño (2016 p.179) las competencias se pueden categorizar de dos formas, la primera está en el comportamiento del empleado en cuanto al nivel de desempeño, la competencia laboral general y en segundo lugar el desempeño o la calidad del trabajo realizado. Esta idea compara la inteligencia individual frente al conocimiento del tema, la habilidad específica frente a la destreza en general y las actitudes hacia las ideas frente a los comportamientos de los empleados. Para definir las competencias de la fuerza laboral, uno debe reconocer las necesidades de los consumidores, los aspectos de marketing y el consumo general del producto vendido o ofrecido. Las competencias que las personas necesitan demostrar y poseer para lograr sus objetivos personales han evolucionado para ser más complejas, incluida la capacidad de cambiar y adaptarse a las tecnologías, analizar y sintetizar grandes cantidades de información recibida.

El trabajo en grupo no puede funcionar si no existe una comunicación adecuada entre los miembros del grupo. Para lograr un trabajo en grupo ideal, los participantes deben cooperar o colaborar para ver esfuerzos fructíferos. En una investigación adicional realizada por Nowak en 2006, el autor cree que, en un mundo global, la cooperación es la clave del éxito. Investigadores adicionales explican que el rendimiento y la innovación del equipo se pueden mejorar con esfuerzos colaborativos. Hay muchos factores adicionales que pueden contribuir a la mentalidad grupal, que incluyen la motivación, el respeto y la comprensión mutuos, los estilos de comunicación y las experiencias previas. Janutaite (2015).

Otro conjunto importante de habilidades son las habilidades de liderazgo. Estos son comportamientos y capacidades que se cree que ayudan a los empleados que desean liderar o actualmente lideran a promover el bienestar de sus empleados y ayudar a otras personas a alcanzar su máximo potencial dentro de la empresa. Estas habilidades pueden verse como acciones enfocadas con la intención de motivación, orientación a metas y cumplimiento de objetivos. El autor Chobharkar en 2011 creó una lista de 5 competencias principales para un líder exitoso. Sus ideas incluyen la planificación que incluye el establecimiento de metas y la planificación de crisis, la formación de equipos que controla el desempeño del grupo y la gestión de 
Competencias educativas en la modalidad virtual docentes, discentes, y el mundo laboral

conflictos, la toma de decisiones que requiere visión, coraje y conocimiento, relaciones que incluyen habilidades de tutoría y fuertes habilidades interpersonales, y finalmente visión y estrategia que incluyen la capacidad para influir y tomar decisiones. Kobicheva (2021).

Al explorar las ideas de las competencias de la fuerza laboral, es importante ver la necesidad no solo del conocimiento, sino también de la habilidad de aprender. No es suficiente ser inteligente en el lugar de trabajo, sino también capacitado para aprender habilidades e información adicionales en el proceso. La idea de desarrollar habilidades que mejoren el trabajo en grupo y la colaboración y cooperación y permitir que estas habilidades se manifiesten a nivel global puede crear un nuevo punto de vista de éxito. La capacidad de liderar, incluso cuando no está en una posición de liderazgo, puede crear un empleado que tenga éxito en la toma de decisiones, los movimientos estratégicos y sea agradable con todos. Todas estas habilidades se alinean directamente con los objetivos de la UNESCO de enriquecer a los estudiantes y futuros trabajadores para prepararlos para la fuerza laboral y sus contribuciones a la sociedad; haciéndolos rentables para sí mismos, pero también haciéndolos candidatos viables para cualquier puesto que deban elegir para seguir creando una fuerza laboral más fuerte y eficiente de empleados respetables.

\section{CONCLUSIONES}

Como se ha estudiado a lo largo de este ensayo, el rol de los docentes nos exige capacitarnos, actualizarnos y estar informados sobre los avances en materia de educación. Así mismo interesarnos en los adelantos tecnológicos $\mathrm{y}$, en la medida de lo posible, adaptar nuevas soluciones para el aprendizaje. Colaborar con otros profesores, compartir ideas y experien cias es fundamental para enriquecer nuestro
Nagari, B y cols.

trabajo y apoyar a nuestros alumnos a mejorar el desarrollo de competencias. Se requieren docentes que impacten positivamente la vida de los alumnos y los formen para que sepan cómo actuar en diferentes situaciones, para que sepan valorarse ellos mismos su desenvolvimiento y sepan tomar decisiones en diferentes contextos. Ahora la educación está formando competencias para la vida y para el aprendizaje permanente de nuestros estudiantes.

Dentro de la revisión realizada se pudo identificar la relevancia que tiene para los aprendices contar con docentes virtuales que aparezcan en las pantallas demostrando de esta manera que es una persona de fácil trato, que proporciona comprensión, paciencia y quien se encuentra apasionado por su curso. Es de vital importancia entonces, que el docente se preocupe por incluir en su enseñanza actividades como la socialización de experiencias de vida, el establecimiento de relaciones de amistad y camaradería en el grupo, dar paso al humor, así como una oportuna revisión de las asignaciones dadas y claridad en la organización del discurso. Alamri y Tyler-Wood, (2017) dicen que "los instructores a distancia requieren algo más que dominio tecnológico; necesitan ser capaces de comunicarse y comprometerse con los estudiantes usando una variedad de recursos". El desarrollo de competencias en los estudiantes es un proceso que requiere tanto de la motivación intrínseca como de la extrínseca para que se dé de forma natural, edificante y constante. Por esto, es importante que los aprendices reciban oportunidades de aprendizaje académico y práctico en distintas disciplinas, al tiempo que tengan la oportunidad de elegir caminos y alternativas a través de las cuales encuentren su pasión y propósito de aprendizaje, lo que pueda redundar en éxito académico y a largo plazo laboral. 
Los estudiantes como la sociedad del futuro nos hacen reflexionar con situaciones cada vez más complejas como es formar para una sociedad más justa y democrática, preparar a nuestros alumnos para desenvolverse en una sociedad que cambia muy rápidamente, contribuir a formar ciudadanos conscientes, responsables y con valores.

\section{REFERENCIAS BIBLIOGRÁFICAS}

Alamri, A., y Tyler-Wood, T. (2017). Factors Affecting Learners with Disabilities- Instructor Interaction in Online Learning. Journal of Special Education Technology, 32(2),59-69. DOI: https://doi.org/10.1177/0162643416681497

Aristizábal Montes, M., Rivera González, R., Bermúdez Bedoya, J.F., \& García Castro, L.I. (2016). Aprender a Aprender en un Modelo de Competencias Laborales. Zona Próxima, (25), 1-21 https://doi.org/10.14482/zp.22.5832

Arteaga Valdés, E., Armada Arteaga, L., \& Del Sol Martínez, J. L. (2016). La enseñanza de las ciencias en el nuevo milenio. Retos y sugerencias. Revista Universidad y Sociedad, 8(1), 169-176 http://scielo.sld.cu/scielo.php?script=sci

Cariaga, R. (2019). El rol del docente universitario en las propuestas educativas virtualizadas.Revista Pilquen. Sección Psicopedagogía, 16(2), 1428. http://revele.uncoma.edu.ar/htdoc/revele/index.php/psico/article/viewFile/2547/59300

Cepeda Romero, O., Gallardo Fernández, I. M., \& Rodríguez Rodríguez, J. (2017). La evaluación de los materiales didácticos digitales. Revista Latinoamericana de Tecnología Educativa, 2017, vol. 16, núm. 2, p. 79-95. DOI: https://doi.org/10.17398/1695-288X.16.2.79

de Valderrama, E. P., \& Torrealba, L. (2020). Los entornos virtuales de aprendizaje (EVA). Una Estrategia Didáctica para la administración de unidades curriculares universitarias. Revista Electrónica de Divulgación de Metodologías emergentes en el desarrollo de las STEM, 1(2), 18-27 http://www.revistas.unp.edu.ar/index.php/rediunp/article/view/119

García-Cabrero, B., Luna-Serrano, E., Ponce-Ceballos, S., Cisneros-Cohenour, E., Cordero-Arroyo, G., Espinoza-Díaz, Y., \& García-Vigil, M. H. (2018). Las Competencias docentes en entornos virtuales: Un modelo para su evaluación. Revista Iberoamericana de Educación a Distancia, 21(1), 343-365. DOI: http://dx.doi.org/10.5944/ried.21.1.18816

George Reyes, C. E., Ramírez Martinell, A. (2019). Competencias Investigativas y Saberes Digitales de Estudiantes de Posgrado en la Modalidad Virtual. Certiuni Journal, (5), 65-78 https://www.researchgate.net/publication/338345883 
Guevara, C. Y., Rugerio, J. P., Hermosillo, A. M. \& Corona, L. A. (2020). Aprendizaje Socioemocional en Preescolar: Fundamentos, Revisión de Investigaciones y Propuestas. Revista Electrónica de Investigación Educativa, 22(26), 1-14 https://doi.org/10.24320/redie.2020.22.e26.2897

Instituto Nacional de Tecnologías Educativas y Formación del Profesorado. Ministerio de Educación, Cultura y Deporte del Gobierno de España. (2017). Marco Común de la Competencia Digital. http://aprende.intef.es/mccdd

Janutaite, S., Vosyliute, V., Vizgirdaite, J., \& Taras, V. (2015). Cross-cultural Virtual Group Work: Cooperation vs. Collaboration (Case of Project 'X-Culture'). Social Sciences, 88(2). https://doi.org/10.5755/j01.ss.88.2.12739

Kobicheva, A. (2021). Virtual project as a tool for leadership skills development. E3S Web of Conferences, 284, 09019. https://doi.org/10.1051/e3sconf/202128409019

Morales Mantilla, S. M. (2011). La Construcción de Competencias en Ambientes Virtuales de Aprendizaje. Revista de Investigaciones UNAD, 10(2), (julio - diciembre), 9-23 https://doi.org/10.22490/25391887.751

Moreno Almazán, O. (2015). Evaluación de la modalidad de interacción de la tutoría y los efectos en el logro académico en entornos en línea. RIED: Revista Iberoamericana de Educación a Distancia.

http://e-spacio.uned.es/fez/eserv/bibliuned:revistaRied-2015-18-1-7110/evaluacion_modalidad.pdf

Organización de las Naciones Unidas para la Educación, la Ciencia y la Cultura. (2015).

Replantear la Educación: ¿hacia un bien común mundial? http://unesdoc.unesco.org/images/0023/002326/232697s.pdf

Ramírez-Montoya, M. S. (2019). Informe técnico de los Proyectos de la Estancia Internacional UNESCO 2019. N/A. DOI: http://hdl.handle.net/11285/636104

Reiban, E. (2018). Las Competencias Investigativas del Docente Universitario. Universidad y Sociedad, 10(4), 75-84 https://doi.org/10.33936/cognosis.v5i3.2351

Ríos Muñoz, D. \& Herrera Araya, D. (2017) Los Desafíos de la Evaluación por Competencias en el Ámbito Educativo. Educação e Pesquisa, 43(4), 1073-1086 https://doi.org/10.1590/S1678-4634201706164230 
Soto, D. M. N., \& Bonilla, M. D. A. El portafolio electrónico como instrumento de evaluación de desempeño, una experiencia en la formación docente.

https://posgradoeducacionuatx.org/pdf2018/A135.pdf

UNESCO. (2020, 14 enero). UNESCO and Sustainable Development Goals. https://en.unesco.org/sustainabledevelopmentgoals

Valdez-Esquivel, W.E., Pérez-Azahuanche, M. A. (2021) Las Competencias Comunicativas como Factor Fundamental para el Desarrollo Social. Polo del Conocimiento 56, 6(3), 433456 DOI:10.23857/PC.V6I3.2380

Valenzuela, A. (2019). ¿Qué hay de nuevo en la Metacognición? Revisión del concepto, sus componentes y términos afines. Educação e Pesquisa 45(5), 1-20 http://dx.doi.org/10.1590/S1678-4634201945187571

Vera Sagredo, A., Poblete Correa, S. \& Días Larenas, C. (2019). Percepción de estrategias y estilos de aprendizaje en estudiantes universitarios de primer año. Revista Cubana de Educación Superior, 38(1), http://scielo.sld.cu/scielo.php?script=sci

Zermeño Padilla, D. A. M., \& Lozano Rodríguez, D. A. (2016). Desarrollo de competencias interpersonales en ambientes virtuales. CPU-e, Revista de Investigación Educativa, 22, 176-199. https://doi.org/10.25009/cpue.v0i22.1946 